\title{
A Variant of the Mountain Pass Theorem and its Application to Hammerstein Integral Equations
}

\author{
V. B. Moroz and P. P. Zabrejko
}

\begin{abstract}
A new variant of the mountain pass theorem based on a 'strong' deformation lemma is presented. Some applications to the existence of non-trivial solutions of nonlinear Hammerstein integral equations are given too.

Keywords: Critical point theory, mountain pass lemma, Hammerstein integral equations, nontrivial solutions
\end{abstract}

AMS subject classification: 58 E 05,45 G 10, 47 H 30

\section{Introduction}

Let $X$ be a Banach space and $\varphi$ a continuously differentiable functional defined on $X$ (i.e. $\varphi \in C^{1}(X, \mathbb{R})$ ) and satisfying the Palais-Smale condition

$(\mathcal{P S})$ Each sequence $\left\{x_{n}\right\}_{n \geq 1} \subset X$ such that $\left|\varphi\left(x_{n}\right)\right| \leq c(n \in \mathbb{N})$ and $\nabla \varphi\left(x_{n}\right) \rightarrow 0$ as $n \rightarrow+\infty$ has a convergent subsequence.

The classical Ambrosetti-Rabinowitz mountain pass lemma [1] states the following:

If $x \in X$ is a point of strict local minimum of a functional $\varphi$ as above, and there exists a point $y \neq x$ such that $\varphi(y) \leq \varphi(x)$, then $\varphi$ has a critical value $c>\varphi(x)$.

This mountain pass lemma is rather simple but it makes it possible to get numerous results on the existence of non-trivial solutions for various classes of nonlinear problems (see, for example, [17]). The present paper is devoted to functionals of special type.

Let us call a functional $\varphi$ quasi-decreasing on $X$ if $\varphi$ is decreasing on each finitedimensional subspace $\tilde{X} \subseteq X$ or, in other words, $\varphi(x) \rightarrow-\infty$ as $\|x\| \rightarrow+\infty \quad(x \in \tilde{X})$.

V. B. Moroz: Belgosuniv., Mekh-Mat. Fak., Pr. Skoriny 4, BY - 220050 Minsk, Belarus P. P. Zabrejko: Belgosuniv., Mekh-Mat. Fak., Pr. Skoriny 4, BY - 220050 Minsk, Belarus

The investigation is partially supported by the International Soros Science Education Program and the Belorussian Fond of Fundamental Investigations:

The authors express their thanks to their friends and colleagues Jürgen Appell, Andrej Lebedev, Sergej Rogozin and Alfonso Vignoli for several helpful and stimulating conversations. The authors thank the Ruhr University at Bochum where this article was finished for kind hospitality and financial support. 
The mountain pass lemma says that each quasi-decreasing functional $\varphi$ having a point $x$ of strict local minimum admits a critical value $c>\varphi(x)$.

Rabinowitz [16] proved by means of the topological notion of linking a general mountain pass lemma; in the case of quasi-decreasing functionals this lemma can be formulated (see [17: Theorem 5.3 and Remark 5.5]) in the following form.

Theorem 1. Let $X=V^{-} \oplus V^{+}$, where $\operatorname{dim} V^{-}<+\infty$, and let $\varphi \in C^{1}(X, \mathbb{R})$ be a quasi-decreasing functional satisfying the $(\mathcal{P} S)$-condition. Suppose, moreover, that

$$
\inf _{\|x\|=\rho} \varphi(x)>0 \quad\left(x \in V^{+}\right)
$$

for some $\rho>0$ and.

$$
\varphi(x)<0 \quad\left(0 \neq x \in V^{-}\right)
$$

Then $\varphi$ has a critical value $c>0$.

This result by Rabinowitz was revised and generalized in different directions by numerous authors (see, for example, $[3-6,17]$ and others). However, the "geometrical" conditions (1) - (2) were unchanged for the case of quasi-decreasing functionals. At the same time it is known that, in the case when the functional $\varphi$ is odd, condition (2) in the "symmetric" mountain pass lemma can be omitted (see [1] and [17: Theorem 9.12]). Under these conditions the functional $\varphi$ has an unbounded sequence of critical values $c_{n}>0 \quad(n \geq 1)$. In this situation one can assume that condition (2) in Theorem 1 about the negative definiteness of the functional $\varphi$ on the whole subspace $V^{-}$, which leads in applications to serious restrictions for the non-linearities involved, is of technical character and can be weakened. The first result in this direction for quasi-decreasing functionals of special type was obtained by Benci (see [5: Theorem 7.12]) with the help of a special variant of the Morse-Conley theory. Recently a new variant of the mountain pass theorem for quasi-decreasing functionals was presented in [11] on the base of the local linking principle. In these variants the "global" condition (2) is replaced by a "local" condition of the type

$$
\varphi(x)<0 \quad\left(0<\|x\| \leq \rho, x \in V^{-}\right)
$$

The present paper is concerned with a new general theorem on critical values for quasidecreasing functionals. Its proof uses a variant of critical point theory based on a "strong" deformation lemma (compare Lemma 1 below and [17: Lemma A.4]) and does not use the standard minimax approach. Namely, a "strong" deformation lemma allows us to take into account finer, homotopy characteristics of the Lebesgue sets of the functional $\varphi$. An analog of the mountain pass theorem from [11] turns out to be a consequence of the main theorem. As applications we consider non-trivial solutions to Hammerstein integral equations with superquadratic non-linearities. 


\section{Preliminaries}

Let $X$ be an infinite dimensional Hilbert space with scalar product $\langle\cdot, \cdot\rangle$ and norm $\|\cdot\|=\langle\cdot, \cdot\rangle^{\frac{1}{2}},\left\{e_{n}\right\}_{n \geq 1}$ an orthonormal basis in $X$, and $X_{n}=\operatorname{Span}\left\{e_{1}, \ldots, e_{n}\right\}$. Further, let $\varphi \in C^{1}(X, \mathbb{R})$ be a functional on $X$ and $\varphi_{n}=\varphi_{\mid X_{n}}$ its restriction to $X_{n}$. The functional $\varphi$ is said to satisfy the $(\mathcal{P S})^{*}$-condition if for each sequence $\left\{x_{n}\right\}_{n \geq 1} \subseteq X$ such that

$$
\varphi\left(x_{n}\right) \leq c, \quad x_{n} \in X_{m(n)}, \quad \lim _{n \rightarrow \infty} \nabla \varphi_{m(n)}\left(x_{n}\right)=0
$$

(for some subsequence of integers $m(n) \uparrow+\infty$ ) there exists a convergent subsequence. It is easy to see that in case of the $(\mathcal{P S})^{*}$-condition each convergent subsequence of the sequence $\left\{x_{n}\right\}_{n \geq 1}$ converges to a critical point of $\varphi$; besides, the $(\mathcal{P S})^{*}$-condition implies the standard Palais-Smale condition (see [11]).

Below the closed and open Lebesgue sets of a functional $\varphi$ with $c \in \mathbb{R} \cup\{+\infty\}$ are denoted by

$$
\varphi^{c}=\{x \in X: \varphi(x) \leq c\} \quad \text { and } \quad \dot{\varphi}^{c}=\{x \in X: \varphi(x)<c\}
$$

respectively; it is natural to put $\varphi^{+\infty}=\dot{\varphi}^{+\infty}=X$.

Let $A \subseteq B \subseteq X$. A deformation of the set $B$ is a continuous (with respect to both variables) mapping $h_{\mathrm{\imath}}:[0,1] \times B \rightarrow B$ such that $h_{0}(x)=x$ for $x \in B$. A set $B$ is deformable into $A$ if there exists a deformation $h_{t}$ of the set $B$ such that $h_{1}(B) \subseteq A$. In the case when $A=\{p\}$, where $p$ is a point in $B, B$ is deformable in itself.

The set $A$ is a deformation retract of $B$ if there is a deformation $h_{t}$ of the set $B$ such that $h_{1}(B) \subseteq A$ and $h_{1}(x)=x$ for all $x \in A$. The set $A$ is called a strong deformation retract of $B$, if $A$ is a deformation retract and, moreover, $h_{t}(x)=x$ for all $x \in A$ and $t \in[0,1]$. It is well known that, if $A$ is a (strong) deformation retract of $B$, then $A$ and $B$ have the same homotopy type. The converse does not hold in general (see, however, Lemma 2 below).

For $C^{1}$-functionals satisfying the $(\mathcal{P S})$-condition the following deformation lemma is well-known (for a proof see, e.g., [7: Theorem 3.2]).

Lemma 1. Let $\varphi \in C^{1}(X, \mathbb{R})$ satisfy the $(\mathcal{P S})$-condition. Assume that the interval $[a, b] \subseteq \mathbb{R} \cup\{+\infty\}$ does not contain critical values of $\varphi$. Then the Lebesgue set $\varphi^{a}$ is a strong deformation retract of both $\varphi^{b}$ and $\dot{\varphi}^{b}$.

We now indicate some elementary consequences of Lemma 1.

Proposition 1. Let $\varphi \in C^{1}(X, \mathbb{R})$ satisfy the $(\mathcal{P S})$-condition. Let $[a, b] \subseteq \mathbb{R} \cup$ $\{+\infty\}$ and assume that the Lebesgue set $\varphi^{a}$ is not a strong deformation retract of $\varphi^{b}$. Then $\varphi$ has a critical value $c \in[a, b]$.

Proposition 2. Let $\varphi \in C^{1}(X, \mathbb{R})$ satisfy the $(\mathcal{P S})$-condition. Let $a \in \mathbb{R}$ be such that the Lebesgue set $\varphi^{a}$ is not deformable in itself to a point. Then $\varphi$ has a critical value $c \geq a$.

In the sequel it will be useful for us to distinguish a particular class of critical values, obtained by Propositions 1 and 2. More exactly, a value $c \in \mathbb{R}$ of the functional $\varphi$ is 
called an essential critical value if there exist arbitrarily small numbers $\varepsilon>0$ such that the Lebesgue set $\varphi^{c-\varepsilon}$ is not a strong deformation retract of the Lebesgue set $\varphi^{c+e}$. From Lemma 1 it follows that each essential critical value of a $C^{1}$-functional $\varphi$ satisfing the $(\mathcal{P S})$-condition is indeed a critical value of $\varphi$. The notion of essential critical values is in fact a topological one and can therefore be extended to continuous functionals. Close notions for continuous functionals in metric spaces were introduced and studied in [8]. The notion of essential critical value is also used in [13).

\section{The main result}

Now we are in a position to present a general mountain pass theorem for quasi-decreasing functionals.

Theorem 2. Let $\varphi \in C^{1}(X, \mathbb{R})$ be a quasi-decreasing functional satisfying the $(\mathcal{P S})^{*}$-condition. Further, let $\varphi$ have an essential critical value $c \in \mathbb{R}$. Then there exist at least two different critical values of $\varphi$.

In order to prove Theorem 2 we need information about the homotopy types of the Lebesgue sets for a functional $\varphi$ in a neighbourhood of an essential critical value and the "asymptotic" homotopy type of the Lebesgue sets $\varphi^{c}$ for $c \rightarrow-\infty$.

We recall that a metric space $C$ is called an absolute neighbourhood retract if for any closed subset $A \subseteq B$ of any metric space $B$ each continuous mapping $f: A \rightarrow C$ can be extended to a neighbourhood of $A$ in $B$. A Banach manifold (with boundary) and, therefore, the Lebesgue set corresponding to a regular value of a $C^{1}$-functional $\varphi$ is an absolute neighbourhood retract (see [14: Theorem 6]).

It is a crucial property for us that the classes of deformation retracts and strong deformation retracts for absolute neighbourhood retracts coincide. In fact, the following statement holds (see [15: p. 90]).

Lemma 2. Let $B$ be an absolute neighbourhood retract and $A \subseteq B$ be a closed subspace of $B$ which is an absolute neighbourhood retract, too. Then $\bar{A}$ is a strong deformation retract of $B$ if and only if the inclusion $i: A \hookrightarrow B$ is a homotopy equivalence.

A simple corollary of Lemma 2 gives us the necessary information on the homotopy type of the Lebesgue sets for a functional $\varphi$ in a neighbourhood of an essential critical value.

Lemma 3. Let $\varphi \in C^{1}(X, \mathbb{R})$ satisfy the $(\mathcal{P S})$-condition. Further, let $c \in \mathbb{R}$ be an isolated and essential critical value of $\varphi$. Then there exist arbitrarily small numbers $\varepsilon>0$ such that at least one of the two Lebesgue sets $\varphi^{c-\varepsilon}$ and $\varphi^{c+\varepsilon}$ is not deformable in itself.

Proof. Fix $\varepsilon>0$ in such a way that the interval $[c-\varepsilon, c+\varepsilon]$ does not contain critical values of $\varphi$ different from $c$ and the Lebesgue set $\varphi^{c-\varepsilon}$ is not a strong deformation retract of the Lebesgue set $\varphi^{c+\varepsilon}$. Since $c+\varepsilon$ and $c-\varepsilon$ are regular values of $\varphi$, the Lebesgue sets $\varphi^{c-e}$ and $\varphi^{c+\varepsilon}$ are closed absolute neighbourhood retracts and, by Lemma 2, we have that the inclusion $i: \varphi^{c-\epsilon} \hookrightarrow \varphi^{c+e}$ is not a homotopy equivalence. Assume now that both $\varphi^{c-c}$ and $\varphi^{c+e}$ are deformable in itself. In this case any continuous map 
$f: \varphi^{c-\varepsilon} \rightarrow \varphi^{c+e}$ and, in particular, the inclusion map is a homotopy equivalence [15: p. 89], which is a contradiction

Now we discuss an auxiliary statement on functionals defined on finite-dimensional spaces.

Lemma 4. Let $\operatorname{dim} \tilde{X}=n<\infty$ and $\psi \in C^{1}(\tilde{X}, \mathbb{R})$ be a decreasing functional having a finite number of critical values. Then for all sufficiently small $a \in \mathbb{R}$, the open Lebesgue set $\dot{\psi}^{a}$ has the homotopy type of the $(n-1)$-dimensional sphere $\mathrm{S}^{n-1}$.

Proof. Fix a number $a \in \mathbb{R}$ such that the functional $\psi$ has no critical values in $(-\infty, a)$. Under the above conditions, $\psi$ attains its maximum on $\tilde{X}$ at a point $x_{0}$. It is easy to see that $x_{0} \notin \varphi^{a}$. Denote by $D_{R}=\tilde{X} \backslash \dot{B}_{R}\left(x_{0}\right)$ the complement to the open ball $\dot{B}_{R}\left(x_{0}\right)$ in the space $\tilde{X}$ with center at the point $x_{0}$ and radius $R>0$. Since $D_{R}$ has the homotopy type of the $(n-1)$-dimensional sphere $S^{n-1}$, the statement of Lemma 4 will be proved if we show that $\dot{\psi}^{a}$ is deformable in $D_{R}$ and $D_{R}$ is a retract of $\dot{\psi}^{a}$, i.e. there exists a continuous mapping $r: \dot{\psi}^{a} \rightarrow D_{R}$ such that $r(x)=x \quad\left(x \in D_{R}\right)$. Then (see [15: p. 84]) $D_{R}$ is a deformation retract of $\dot{\psi}^{a}$ and, in particular, $\dot{\psi}^{a}$ and $D_{R}$ have the same homotopy type.

Since $\psi$ is decreasing on $X$, there exist a number $b<a$ and a sufficiently large number $R$ such that the embeddings $\psi^{b} \hookrightarrow D_{R} \hookrightarrow \dot{\psi}^{a}$ hold. Further, since $\psi$ does not have critical values in the interval $(-\infty, a]$, by virtue of Lemma 1 the Lebesgue set $\psi^{b}$ is a strong deformation retract of $\dot{\psi}^{a}$. In particular, $\dot{\psi}^{a}$ is deformable in $D_{R}$.

Define a retraction $r: \dot{\psi}^{a} \rightarrow D_{R}$ by means of the formula

$$
r(x)= \begin{cases}x_{0}+R\left\|x-x_{0}\right\|^{-1}\left(x-x_{0}\right) & \text { if } x \in \dot{\psi}^{\alpha} \backslash D_{R} \\ x & \text { if } x \in D_{R} .\end{cases}
$$

Since $x_{0} \notin \dot{\psi}^{a}$, the mapping $r$ is continuous, and $r(x) \in D_{R}$ for all $x \in \dot{\psi}^{a}$. Moreover, by definition, $r(x)=x$ for all $x \in D_{R}$. Thus, $r$ is indeed a retraction of $\dot{\psi}^{a}$ to $D_{r}$

Let us recall some definitions. Let $\left\{X_{n}\right\}_{n \geq 1}$ be an increasing sequence of topological spaces $\left(X_{n} \subseteq X_{n+1}\right)$ and $X_{\infty}=\cup_{n \geq 1} X_{n}$ their union. The set $X_{\infty}$ equipped with the finest topology in which all embeddings $i_{n}: X_{n} \hookrightarrow X_{\infty}$ are continuous is called the inductive limit of the sequence $\left\{X_{n}\right\}_{n \geq 1}$; it is denoted by $X_{\infty}=\operatorname{liminj} X_{n}$. The following lemma is one of the fundamental results in infinite-dimensional homotopy theory (see [14: Corollary of Theorem 17]).

Lemma 5. Let $U \subseteq X$ be an open set of $X$ and $U_{\infty}=\operatorname{liminj}\left(U \cap X_{n}\right)$. Then the embbeding $i: U_{\infty} \hookrightarrow U$ is a homotopy equivalence.

Using Lemmas 4 and 5 one can easily calculate the "asymptotic" homotopy type of the Lebesgue sets of a quasi-decreasing functional as $c \rightarrow-\infty$.

Lemma 6. Let $\varphi \in C^{1}(X, \mathbb{R})$ be a quasi-decreasing functional satisfying the $(\mathcal{P} S)^{*}$ condition. Assume that $\varphi$ has only a finite number of critical values. Then for all sufficiently small $a \in \mathbb{R}$ the Lebesgue set $\varphi^{a}$ is deformable in itself.

Proof. Fix a number $a \in \mathbb{R}$ such that the functional $\psi$ has no critical values in $(-\infty, a]$. Since $\varphi$ satisfies the $(\mathcal{P S})^{*}$-condition, it is easy to see that the functionals $\varphi_{n}$ 
have no critical values in the interval $(-\infty, a]$ for all sufficiently large $n$. Moreover, the functionals $\varphi_{n}$ are decreasing on $X_{n}$. Further, Lemma 4 implies that the open Lebesgue set $\dot{\varphi}_{n}^{a}=\dot{\varphi}^{a} \cap X_{n}$ has the homotopy type of the sphere $\mathbb{S}^{n-1}$. Correspondingly, the inductive limit $\dot{\varphi}_{\infty}^{a}=\operatorname{liminj} \dot{\varphi}_{n}^{a}$ has the homotopy type of the sphere $\mathbf{S}^{\infty}$.

Now, due to Lemma 5 the embedding $i: \dot{\varphi}_{\infty}^{a} \hookrightarrow \dot{\varphi}^{a}$ is a homotopy equivalence. But this means that the open Lebesgue set $\dot{\varphi}^{a}$ has the homotopy type of the sphere $\mathbb{S}^{\infty}$, and therefore is deformable in itself. Further, according to Lemma 1 , for each $b<a$ the Lebesgue set $\varphi^{b}$ is a strong deformation retract of the open Lebesgue set $\dot{\varphi}^{a}$, and hence deformable in itself, too $\mathbf{n}$

Notice that the $(\mathcal{P S})^{*}$-condition was used only in the proof of Lemma 6 . In order to prove other lemmas the "standard" $(\mathcal{P S})$-condition is sufficient.

\section{Proof of the main result and further ones}

First let us now prove our main result.

Proof of Theorem 2. We start with assuming that $\varphi$ has a finite number of critical values, for otherwise the theorem is proved. Let $c \in \mathbb{R}$ be an essential critical value of $\varphi$. By virtue of Lemma 3 there exists an arbitrarily small number $\varepsilon>0$ such that at least one of the two Lebesgue sets $\varphi^{c+\varepsilon}$ and $\varphi^{c-\varepsilon}$ is not deformable in itself. First assume that the set $\varphi^{c+\varepsilon}$ is not deformable in itself. Then Proposition 2 implies that $\varphi$ has a critical value $c_{1}>c$.

Now assume that $\varphi^{c-e}$ is not deformable in itself. Then, by Lemma 6 , for all sufficiently small $a \in \mathbb{R}$ the Lebesgue set $\varphi^{a}$ is deformable in itself. On the other hand, $\varphi^{a}$ cannot be a strong deformation retract of the non-deformable set $\varphi^{c-\varepsilon}$. Thus, by Proposition 1 , the functional $\varphi$ has a critical value $c_{1}<c \boldsymbol{\theta}$

The assertion of Theorem 2 is rather general and therefore not convenient in appli-. cations to nonlinear problems. But it is evident that any condition which guarantees the existence of an essential critical value for the given functional $\varphi$ implies a corresponding variant of the mountain pass lemma.

Let us present some examples.

Proposition 3. Let $\varphi \in C^{1}(X, \mathbb{R})$ satisfy the $(\mathcal{P S})$-condition and $x$ be a point of strict local minimum for $\varphi$. Then $c=\varphi(x)$ is an essential critical value of $\varphi$.

Proposition 4. Let $X=V^{-} \dot{\oplus} V^{+}$, where $\operatorname{dim} V^{-}<\infty$, and let $\varphi \in C^{1}(X, \mathbb{R})$ satisfy the $(\mathcal{P S})$-condition. Assume that $\varphi(0)=0$ and, for each $\rho>0$ sufficiently small,

$$
\inf _{\|x\|=\rho} \varphi(x)>0 \quad\left(x \in V^{+}\right) \quad \text { and } \quad \varphi(x)<0 \quad\left(0<\|x\| \leq \rho, x \in V^{-}\right) .
$$

Then $c=0$ is an essential critical value of $\varphi$.

The proofs of Propositions 3 and 4 are standard (see, for example, [12]). It should be noted that it is necessary to use the definition of essential critical value based on the notion of strong deformation retraction in order to prove Proposition 4. 
One can formulate more general conditions for the existence of an essential critical value on the base of linking conditions of different types (see $[11,12,17]$ ).

The classical mountain pass lemma of Ambrosetti-Rabinowitz is a union of Theorem 2 and Proposition 3. An analogous union of Theorem 2 and Proposition 4 allows us to formulate the following variant of the mountain pass lemma in which only local geometrical conditions are utilized.

Theorem 3. Let $X=V^{-} \oplus V^{+}$, where $\operatorname{dim} V^{-}<\infty$, and let $\varphi \in C^{1}(X, \mathbb{R})$ be a quasi-decreasing functional satisfying the $(\mathcal{P S})^{*}$-condition. Assume that $\varphi(0)=0$ and, for each sufficiently small $\rho>0$,

$$
\inf _{\|x\|=\rho} \varphi(x)>0 \quad\left(x \in V^{+}\right)
$$

and

$$
\varphi(x)<0 \quad\left(0<\|x\| \leq \rho, x \in V^{-}\right) .
$$

Then $\varphi$ has a critical value $c \neq 0$.

Note that, in contrast to Theorem 1 where the existence of a positive critical value is stated, Theorem 3 allows us to prove the existence of critical values of arbitrary sign. As one can see in the proof of Theorem 2, this fact is crucial and is based on the substitution of conditions (1) - (2) by conditions (4) - (5).

\section{Applications}

We apply the results given above to obtain the existence of non-trivial solutions to the nonlinear Hammerstein integral equation

$$
x(t)=\int_{\Omega} k(t, s) f(s, x(s)) d s
$$

where $\Omega \subseteq \mathbb{R}^{m}$ is a bounded domain, $k: \Omega \times \Omega \rightarrow \mathbb{R}$ is a measurable symmetric kernel and the function $f: \Omega \times \mathbb{R} \rightarrow \mathbb{R}$ satisfies the Carathéodory conditions.

Equation (6) can be rewritten in the operator form $x=K F x$, where $F$ is the nonlinear superposition operator

$$
F x(s)=f(s, x(s))
$$

generated by the Carathéodory function $f$ and $K$ is the linear integral operator

$$
K x(t)=\int_{\Omega} k(t, s) x(s) d s
$$

generated by the kernel $k$. In what follows, we assume that $K$ is selfadjoint positive definite and compact as an operator acting in $L_{2}$. In this case its spectrum $\sigma(K)$ consists of a countable set of positive characteristic values $\lambda_{1}>\lambda_{2}>\ldots$ of finite multiplicity, and zero is the only accumulation point. 
Let $\langle\cdot, \cdot\rangle$ and $\|\cdot\|=\langle\cdot, \cdot\rangle^{\frac{1}{2}}$ stand for the scalar product and norm in $L_{2}$, respectively, and let $\varphi: L_{2} \rightarrow \mathbb{R}$ be the Golomb functional generated by $K$ and $F$, i.e.

$$
\varphi(x)=\frac{1}{2}\langle h, h\rangle-\int_{\Omega} \phi(s, H h(s)) \dot{d} s
$$

where $H=K^{\frac{1}{2}}$ is the square root from $K$ and

$$
\phi(s, u)=\int_{0}^{u} f(s, v) d v
$$

is the potential generated by $f$.

Assume that for some $p \in(2, \infty)$ and $p^{\prime}=\frac{p}{p-1}$ the operator $K$ acts and is compact from $L_{p^{\prime}}$ into $L_{p}$. Furthermore, assume that the operator $F$ acts from $L_{p}$ into $L_{p^{\prime}}$; the latter means that the function $f$ satisfies an inequality

$$
|f(s, u)| \leq m(s)+b|u|^{p-1}
$$

with $m \in L_{p^{\prime}}$ and $b<\infty$. Then (see $[9,18]$ ) the Golomb functional is continuously differentiable on the space $L_{2}$ and its gradient has the form

$$
\nabla \varphi(h)=h-\tilde{H} F H h
$$

where $\tilde{H}: L_{p^{\prime}} \rightarrow L_{2}$ is a natural extension of the operator $H$ to $L_{p^{\prime}}$. Moreover, under the conditions above the operator $\tilde{H} F H$ acts in $L_{2}$ and is compact, and each critical point $h^{*} \in L_{2}$ of the Golomb functional $\varphi$ defines a solution $x^{*}=H h^{*} \in L_{p}$ to the Hammerstein equation (6).

Now assume that the function $\phi$ has the form

$$
f(s, u)=a(s) u+w(s, u)
$$

where $a \in L_{\frac{p}{p-2}}$ and $w$ satisfies the special condition that for each $\varepsilon>0$ there exist $a_{\varepsilon} \in L_{\frac{p}{p-2}}$ and $b_{\varepsilon} \in \mathbb{R}^{+}$. such that $\left\|a_{\varepsilon} \mid L_{\frac{p}{p-2}}\right\|<\varepsilon$ and

$$
|w(s, u)| \leq a_{\varepsilon}(s)|u|+b_{\varepsilon}|u|^{p-1}
$$

the latter guarantees that the Golomb functional $\varphi$ is twice differentiable at zero (see $[10,19])$, and

$$
\nabla^{2} \varphi(0)=I-\tilde{H} A H \quad(A x(s)=a(s) x(s))
$$

is its Hessian. Set

$$
\omega(s, u)=\int_{0}^{u} w(s, t) d t .
$$

In what follows we are interested in non-trivial solutions to the Hammerstein integral equation (6) under the assumptions

$$
\omega(s, u) \geq \gamma|u|^{q}-b(s) \quad(s \in \Omega, u \in \mathbb{R})
$$


where $\gamma>0,2<q \leq p$ and $b \in L_{1}$, and

$$
a \in L_{\frac{r}{r-2}} \quad \text { and } \quad \omega(s, u)-\xi u w(s, u) \leq-\delta|u|^{r}+c(s) \quad(s \in \Omega, u \in \mathbb{R})
$$

where $\delta>0,2<r \leq p, \xi<\frac{1}{2}$ and $c \in L_{1}$. Nonlinearities of such type are considered as superquadratic.

A vast literature is devoted to quasilinear elliptic equations with superquadratic nonlinearities (see $[1,4,5,16,17])$. The problem of finding non-trivial solutions to Hammerstein integral equations with non-linearities of such type is considered in [1] under the assumption that $a(s)=a<\lambda_{1}^{-1}$. In this case, 0 is a point of local minimum of the Golomb functional $\varphi$ and the classical mountain pass lemma is applied to this functional. In the case when $a(s)=a \geq \lambda_{1}^{-1}$ one can apply Theorem 1 ; however, to check (2) it is necessary to require in addition that the function $\omega$ is non-negative on $\Omega \times \mathbb{R}$.

Theorem 3 allows us to prove the existence of non-trivial solutions to the Hammerstein equation (6) without assumptions of such type.

Theorem 4. Let the conditions (9)-(10) hold. Moreover, suppose that one of the following conditions is true:

a) $1 \notin \sigma(K A)$.

b) $1 \in \sigma(K A)$ and the condition

$\omega(s, u) \geq a|u|^{p} \quad\left(|u| \leq u_{0}(s)\right) \quad$ and $\quad-\omega(s, u) \leq L|u|^{p} \quad\left(|u|>u_{0}(s)\right)$

holds for some $a>0, L<\infty$ and some measurable positive function $u_{0}$.

c) $1 \in \sigma(K A)$, the eigenfunctions of $K A$ corresponding to eigenvalues from $[1, \infty)$ belong to $L_{\bar{p}}$, and the condition

$$
\omega(s, u) \geq a|u|^{\bar{p}}\left(|u| \leq u_{0}(s)\right) \quad \text { and } \quad-\omega(s, u) \leq L|u|^{\bar{p}}\left(|u|>u_{0}(s)\right)
$$

holds for some $a>0, p<\tilde{p}<\infty, L<\infty$ and some measurable positive function $u_{0}$.

Then the Hammerstein equation (6) has a non-trivial solution in the space $L_{p}$.

\section{Proof of Theorem 4}

In order to prove Theorem 4 it is sufficient to show that the Golomb functional $\varphi$ satisfies the conditions of Theorem 3 on the space $X=L_{2}$. It can be rewritten in the form

$$
\varphi(h)=\frac{1}{2}\langle(I-\tilde{H} A H) h, h\rangle-\psi(h)
$$

where

$$
\psi(h)=\int_{\Omega} \omega(s, H h(s)) d s .
$$

It is obvious that $\psi(h)=o\left(\|h\|^{2}\right)$ as $\|h\| \rightarrow 0$ and

$$
\nabla^{2} \varphi(0)=I-\tilde{H} A H
$$

is the Hessian of the functional $\varphi$ at zero.

Assume that $L_{2}=V^{-} \oplus V^{+}$where $V^{+}$is the positive invariant subspace of the operator $I-\tilde{H} A H$ and $V^{-}$is its orthogonal complement. It is easy to see that $\operatorname{dim} V^{-}<$ $+\infty$. 
Lemma 7. Let Condition (9) be satisfied. Then the Golomb functional $\varphi$ is quasidecreasing on the space $L_{2}$.

Proof. Since the operator $K$ acts from $L_{p^{\prime}}$ into $L_{p}$, the operator $H$ acts from $L_{2}$ into $L_{p} \subseteq L_{q}$ (see, e.g., [10: p. 176]). Further, due to (9) we have

$$
\begin{aligned}
\psi(h) & =\int_{\Omega} \omega(s, H h(s)) d s \geq \gamma \int_{\Omega}|H h(s)|^{q} d s-\left\|b(s) \mid L_{1}\right\| \\
& =\gamma\left\|H h\left|L_{q}\left\|^{q}-\right\| b(s)\right| L_{1}\right\| \quad\left(h \in L_{2}\right) .
\end{aligned}
$$

Now assume that $\tilde{X}$ is a finite-dimensional subspace of $L_{2}$. Then

$$
\left\|H h \mid \dot{L_{q}}\right\| \geq \alpha\|h\| \quad(h \in \tilde{X})
$$

for a positive $\alpha=\alpha(\tilde{X})$. This inequality implies

$$
\psi(h) \geq \alpha^{q} \gamma\|h\|^{q}-\left\|b(s) \mid L_{1}\right\|
$$

and, further,

$$
\varphi(h) \leq\|I-\tilde{H} A H\|\|h\|^{2}-\alpha \gamma^{q}\|h\|^{q}+\left\|b(s) \mid L_{1}\right\| .
$$

Since $q>2$, we obtain $\varphi(h) \rightarrow-\infty$ as $h \in \tilde{X}$ and $\|h\| \rightarrow+\infty$. This means that $\varphi$ is a quasi-decreasing functional on the space $L_{2}$

Lemma 8. Let condition (10) be satisfied. Then the Golomb functional $\varphi$ satisfies the $(\mathcal{P S})^{*}$-condition.

Proof. Assume that $\left\{e_{n}\right\}_{n \geq 1} \subseteq L_{2}$ is an orthonormed basis in the space $L_{2}$ consisting of eigenfunctions of the operator $K$,

$$
X_{n}=\operatorname{Span}\left\{e_{1}, \ldots, e_{n}\right\} \text {, }
$$

and $P_{n}$ are orthoprojectors on the subspaces $X_{n}$. Assume that $\left\{h_{n}\right\}_{n \geq 1} \subseteq L_{2}$ is a sequence such that

$$
\varphi\left(h_{n}\right) \leq c \quad\left(h_{n} \in X_{m(n)}\right) \quad \text { and } \quad \lim _{n \rightarrow \infty} \nabla \varphi_{m(n)}\left(h_{n}\right)=0
$$

for some subsequence of integers $m(n) \uparrow+\infty$. First we show that the sequence $\left\{h_{n}\right\}_{n \geq 1}$ is bounded in $L_{2}$.

Fix $\varepsilon>0$. Then the elementary identity

$$
\begin{aligned}
\left(\frac{1}{2}-\xi\right)\left\|h_{n}\right\|^{2}= & \varphi\left(h_{n}\right)-\xi\left(\nabla \varphi_{m(n)}\left(h_{n}\right), h_{n}\right\rangle \\
& +\left(\frac{1}{2}-\xi\right)\left\langle\tilde{H} A H h_{n}, h_{n}\right)+\psi\left(h_{n}\right)-\xi\left\langle\nabla \psi\left(h_{n}\right), h_{n}\right\rangle
\end{aligned}
$$

implies that the inequality

$$
\begin{aligned}
\left(\frac{1}{2}-\xi\right)\left\|h_{n}\right\|^{2} \leq & c+\varepsilon\left\|h_{n}\right\|+\left(\frac{1}{2}-\xi\right) \beta\left\|H h_{n} \mid L_{r}\right\|^{2} \\
& +\int_{\Omega}\left(\dot{\omega}\left(s, H h_{n}(s)\right)-\xi H h_{n}(s) w\left(s, H h_{n}(s)\right)\right) d s
\end{aligned}
$$


holds with a $\beta<+\infty$ for sufficiently large $n$. Further, by virtue of (10),

$$
\begin{aligned}
\left(\frac{1}{2}-\xi\right)\left\|h_{n}\right\|^{2} & \leq c+\varepsilon\left\|h_{n}\right\|+\left[\left(\frac{1}{2}-\xi\right) \beta\left\|H h_{n}\left|L_{r}\left\|^{2}-\delta \int_{\Omega}\left|H h_{n}(s)\right|^{r} d s+\right\| c(s)\right| L_{1}\right\|\right] \\
& =\varepsilon\left\|h_{n}\right\|+\left[\left(\frac{1}{2}-\xi\right) \beta\left\|H h_{n}\left|L_{r}\left\|^{2}-\frac{\delta}{\mu}\right\| H h\right| L_{r}\right\|^{r}+c+\left\|c(s) \mid L_{1}\right\|\right] .
\end{aligned}
$$

It is easy to see that

$$
m=\sup _{0<t<\infty}\left[\left(\frac{1}{2}-\xi\right) \beta t^{2}-\frac{\delta}{\mu} t^{r}\right]<+\infty
$$

and thus we have

$$
\left(\frac{1}{2}-\xi\right)\left\|h_{n}\right\|^{2} \leq \varepsilon\left\|h_{n}\right\|+m+c+\left\|c(s) \mid L_{1}\right\|
$$

for sufficiently large $n$. Since $\varepsilon$ is fixed, the last inequality implies the boundedness of the sequence $\left\{h_{n}\right\}_{n \geq 1}$ in $L_{2}$.

Finally, by virtue of the relation

$$
\left\|\nabla \varphi_{n(m)}\left(h_{n}\right)\right\|=\left\|h_{n}-P_{m(n)} \tilde{H} F H h_{n}\right\| \rightarrow 0 \quad(n \rightarrow+\infty)
$$

and by the complete continuity of the operator $\tilde{H} F H$ in the space $L_{2}$, the sequence $\left\{h_{n}\right\}_{n \geq 1}$ contains a convergent subsequence

Lemma 9. Let one of conditions a) - c) of Theorem 4 hold. Then there exists $\rho>0$ such that

$$
\psi(h)>0 \quad\left(h \in V^{-}, 0<\|h\| \leq \rho\right) .
$$

Proof. In the case when condition a) holds the statement of the lemma is evident. Let condition b) hold. Then, by virtue of (9),

$$
\begin{aligned}
\int_{\Omega} \omega(s, H h(s)) d s & =\int_{\Omega \backslash D(h)} \omega(s, H h(s)) d s+\int_{D(h)} \omega(s, H h(s)) d s \\
& \geq a \int_{\Omega \backslash D(h)}|H h(s)|^{p} d s-L \int_{D(h)}|H h(s)|^{p} d s \\
& =a \int_{\Omega}|H h(s)|^{p} d s-(a+L) \int_{D(h)}|H h(s)|^{p} d s \\
& =a\left\|\left.H h(s)\left|L_{p} \|^{p}-L \int_{D(h)}\right| H h(s)\right|^{p} d s\right.
\end{aligned}
$$

where $D(h)=\left\{s \in \Omega:|H h(s)|>u_{0}(s)\right\}$. Now we remark that there exists a positive number $\nu$ such that

$$
\left\|H h(s) \mid L_{p}\right\| \geq \nu\|h\| \quad\left(h \in V^{-}\right)
$$

and, furthermore, there exists a function $e \in L_{p}$ such that

$$
|H h(s)| \leq\|h\| e(s) \quad\left(h \in V^{-}\right) .
$$


Applying these inequalities to the previous one we obtain

$$
\int_{\Omega} \omega(s, H h(s)) d s \geq\left(a \nu^{p}-\int_{T(\|h\|)} e(s)^{p} d s\right)\|h\|^{p}
$$

where $T(r)=\left\{s \in \Omega: r e(s)>u_{0}(s)\right\}$. It is evident that mes $T(r) \rightarrow 0$ as $r \rightarrow 0$ and thus the inequality

$$
\int_{\Omega} \omega(s, H h(s)) d s>0
$$

holds for sufficiently small $h \in V^{-}$. The case when condition c) holds is similar; it is sufficient to remark that in this case the operator $H$ acts from $L_{2}$ into $L_{\tilde{p}}$

Now we give the

Proof of Theorem 4. By virtue of Lemmas 7 and 8 , the Golomb functional $\varphi$ is quasi-decreasing on the space $L_{2}$ and satisfies the $(\mathcal{P S})^{*}$-condition. Moreover, by definition of the subspace $V^{+}$, the functional $\varphi$ satisfes condition (4) of Theorem 3 . In order to prove Theorem 4 it is necessary to check condition (5) on the subspace $V^{-}$. The latter follows from Lemma 9

In conclusion we note that Theorem 4 can be generalized to Hammerstein integral equations with quasi-positive definite kernels, as well as to systems of Hammerstein integral equations or, in other terms, Hammerstein integral equations in ideal spaces of vector-functions.

\section{References}

[1] Ambrosetti, A. and P. H. Rabinowitz: Dual variational methods in critical point theory and applications. J. Funct. Anal. 14 (1973), $349-381$.

[2] Appell, J. and P. P. Zabrejko: Nonlinear Superposition Operators. Cambridge: University Press 1990.

[3] Bartolo, P., Benci, V. and D. Fortunato: Abstract critical point theorems and applications to some problems with strong resonance at infinity. Nonlin. Anal. 7 (1982), $981-1012$.

[4] Benci, V.: Some critical point theorems and applications. Comm. Pure Appl. Math. 33 (1980), 147 - 172.

[5] Benci, V.: A new approach to the Morse-Conley theory and some applications. Ann. Mat. Pura Appl. (4) 158 (1991), 231 - 305.

[6] Benci, V. and P. H. Rabinowitz: Critical point theorems for indefinite functionals. Invent. Math. 52 (1979), $241-243$.

[7) Chang, K.C.: Infinite-Dimensional Morse Theory and Multiple Solution Problems. Boston: Birkhäuser Verlag 1993.

[8] Degiovanni, M. and S. Lancelotti: Perturbation of even nonsmooth functionals. Diff. Int. Equ. 8 (1995), 981 - 992.

[9] Krasnosel'skii, M. A.: Topological Methods in the Theory of Nonlinear Integral Equations (in Russian). Moscow: Gostekhizdat 1956. 
[10] Krasnosel'skii, M. A., Zabrejko, P. P., Pustyl'nik, E. I. and P. E. Sobolevskii: Integral Operators in Spaces of Summable Functions (in Russian). Moscow: Nauka 1966.

[11] Li, S. J. and M. Willem: Applications of local linking to critical point theory. J. Math. Anal. Appl. 189 (1995), 6 - 32.

[12] Marino, A., Micheletti, A. M. and A. Pistoia: Some variational results on semilinear problems with asymptotically nonsymmetric behaviour. In: Nonlinear Analysis. A tribute in honour of Giovanni Prodi (eds.: A. Ambrosetti and A. Marino). Quaderno Scuola Norm. Sup. Pisa 1991, pp. 243 - 256.

[13] Moroz, V. B., Vignoli, A. and P. P. Zabrejko: On the Three Critical Points Theorem. Preprint.

[14] Palais, R. S.: Homotopy theory of infinite dimensional manifolds. Topology 5 (1966), 1 16.

[15] Postnikov, M. M.: Lectures on Algebraic Topology. Foundations of Homotopy Theory (in Russian). Moscow: Nauka 1984.

[16] Rabinowitz, P. H.: Some critical point theorems and applications to semilinear partial differential equations. Ann. Scuola Norm. Sup. Pisa (4) 5 (1978), 215 - 223.

[17] Rabinowitz, P. H.: Minimax Methods in Critical Point Theory with Applications to Differential Equations. Reg. Conf. Ser. Math. 65 (1986), 1 - 100.

[18] Zabrejko, P. P. and A. I. Povolotskii: On the theory of Hammerstein eqations (in Russian). Ukrain. Matem. Zhurn. 22 (1970), 150 - 162.

[19] Zabrejko, P. P. and A. I. Povolotskii: On second solutions of Hammerstein equations (in Russian). Vestnik Yaroslavsk. Univ. 2 (1973), 24 - 30. 